\title{
Il faut étudier l'Afrique avec humilité
}

Entretien conduit par Jean-Luc Racine avec la contribution de Laurent Fourchard

Studying Africa: The Need for Humility

Interview with John Lonsdale

John Lonsdale

\section{OpenEdition}

Journals

Édition électronique

URL : http://journals.openedition.org/transcontinentales/464

DOI : 10.4000/transcontinentales.464

ISBN : 978-2-8218-1408-0

ISSN : 1775-397X

Éditeur

Editions de la maison des sciences de l'homme

\section{Édition imprimée}

Date de publication : 30 juin 2006

Pagination : 101-108

ISBN : 2200-92169-1

ISSN : 1950-1684

\section{Référence électronique}

John Lonsdale, « II faut étudier l'Afrique avec humilité », Transcontinentales [En ligne], 2 | 2006, document 7, mis en ligne le 30 septembre 2012, consulté le 07 septembre 2020. URL : http:// journals.openedition.org/transcontinentales/464; DOI : https://doi.org/10.4000/transcontinentales. 464

Ce document a été généré automatiquement le 7 septembre 2020.

Tous droits réservés 


\title{
Il faut étudier l'Afrique avec humilité
}

\author{
Entretien conduit par Jean-Luc Racine avec la contribution de Laurent \\ Fourchard
}

Studying Africa: The Need for Humility

Interview with John Lonsdale

John Lonsdale

\section{NOTE DE L'ÉDITEUR}

John Lonsdale ${ }^{1}$ - jml1001@cam.ac.uk - Fellow de Trinity College depuis 1964, est professeur d'histoire africaine à l'université de Cambridge. Il est l'auteur d'ouvrages de référence sur l'histoire sociale, cultu-relle, religieuse et politique du Kenya (dont Mau Mau and Nationhood: Arms, Authority and Narration, avec Atieno Odhiambo, Oxford et Nairobi, 2003) et a beaucoup travaillé sur les paramètres sociaux et moraux du patriotisme ethnique, sur le nationalisme et sur les communautés religieuses en Afrique. Membre de la Royal African Society, il siège également au conseil scientifique du pôle Afrique des instituts de recherche français à l'étranger. Dans cet entretien accordé à Transcontinentales, ce grand nom de l'africanisme britannique remet en perspective les spécificités de l'histoire coloniale africaine qui aident à comprendre pourquoi les espoirs nourris à l'heure des indépendances ont été en partie déçus. Commentant les représentations convenues des médias et les ambiguïtés des grandes opérations humanitaires en faveur de l'Afrique, John Lonsdale appelle à reconnaître l'impérieuse nécessité d'œuvrer à un meilleur équilibre dans les pratiques de négociations portant sur l'Afrique, et souligne combien la redécouverte de l'histoire africaine pourra contribuer à nourrir une citoyenneté critique.

1 Transcontinentales : Dans un important article publié à l'automne $2005^{1}$, vous rappelez qu'à l'heure des indépendances, sous l'influence des grands dirigeants nationalistes Léopold Sédar Senghor, Patrice Lumumba, Kwame N'Krumah, Jomo Kenyatta ou Julius 
Nyerere - l'espoir prévalait de voir «les Africains, portés par l'énergie de leurs nouvelles souverainetés, terrasser les dragons de la pauvreté, de l'ignorance et de la maladie ». Quarante plus tard, l'image de l'Afrique a changé, et l'optimisme dynamique des années 1960 s'est bien effrité. Qu'est-il arrivé?

2 John Lonsdale : Les processus historiques sont toujours extrêmement complexes. Nous parlons ici de la relation entre des économies et des structures sociales locales et un système commercial globalisé. Cette relation doit être analysée en se référant tour à tour au long terme et au court terme. En ce qui concerne le long terme, on pourrait simplement dire que l'Afrique s'est industrialisée très tardivement par rapport au reste $\mathrm{du}$ monde, et que plus une industrialisation est tardive, plus elle est difficile, plus l'intervention de l'État est nécessaire pour porter attention aux marchés mondiaux, à la gestion des matières premières, à la promotion de l'éducation et à la formation d'une main-d'œuvre qualifiée. Il a donc été très difficile, pour tout État africain qui a choisi cette voie, de s'élever d'une position de producteur de matières premières à celle d'une économie diversifiée, susceptible d'aider la population à échapper aux risques liés à la production de seules matières premières.

3 La difficulté tient aussi à deux spécificités structurelles, la première étant la faiblesse du concept de propriété en Afrique, due sans doute à la faible densité de population favorisant généralement un mode d'accès flexible à la terre. Il était beaucoup plus difficile de trouver en Afrique des propriétés et des biens garantis sur lesquels investir qu'au Japon, en Corée, en Asie du Sud-Est ou en Inde. L'Inde, par exemple, possédait un sens bien plus marqué de la propriété que n'importe quel État africain, ainsi qu'un système financier bien plus développé pour traiter des dettes paysannes depuis des siècles, et c'est pour cela que l'Inde s'est mieux adaptée à la donne industrielle. Rien de tout cela n'existait en Afrique, qui s'est trouvée affectée par le statut incertain de la propriété, et surtout la difficulté à générer du capital et de la sécurité commerciale.

4 Je pense en outre que la faiblesse des États africains doit beaucoup à la faiblesse des États coloniaux qui les ont précédés. C'est une erreur que de penser que les structures coloniales étaient partout puissantes. Elles l'étaient en Afrique du Sud, peut-être en Algérie, mais ailleurs les États coloniaux avaient déployé fort peu de pouvoir idéologique ou coercitif. En comparaison avec l'Europe du XIXe siècle, on pourrait dire que l'Afrique n'a pas connu le nationalisme de la cour d'école.

TC : Le nationalisme de la cour d'école?

6 J. L.: Oui, le nationalisme de la cour de récréation, que la France a cultivé quand les paysans de Bretagne ou du Languedoc apprirent à parler le français à l'école et à l'armée. Vous n'aviez en Afrique ni éducation obligatoire, ni service militaire. On n'y trouve pas ces outils du processus de construction nationale qu'ont connus l'Europe continentale et la Grande-Bretagne au XIX ${ }^{e}$ siècle. Derrière leur image d'oppresseurs et d'alliés du capital international exploitant la main-d'œuvre et les ressources locales, les gouvernements coloniaux exercèrent en fait sur les populations africaines qu'ils dominaient un contrôle bien plus faible, en particulier sur le plan idéologique, que celui qui fut exercé sur les populations européennes à l'âge des nationalismes. Ainsi les États coloniaux africains, relativement faibles, ont généré des mouvements nationalistes tardifs, qui n'héritaient pas de modes de construction nationale comparables à ceux que l'Europe avait connus en termes d'organisation de l'État, d'urbanisation, d'industrialisation de l'économie, de politique linguistique... Ces jeunes États ne contrôlaient que partiellement leur pays, et s'inquiétaient continuellement de leur 
propre sécurité. L'appareil d'État chercha sans cesse des assurances contre d'éventuels désastres, des assurances au rang desquelles on peut ranger aussi bien les loyautés ethniques que les comptes en Suisse. Les élites africaines, marquées par cette incertitude, tentèrent de gouverner des populations au total très fragmentées.

7 TC : Dans vos travaux, vous rappelez que trois modèles ont successivement été élaborés pour tenter d'expliquer les évolutions africaines : la théorie de la modernisation, les théories du sous-développement, et ce que l'on qualifie de néo-patrimonialisme, un modèle qui donne un poids particulier à la résilience créative des paysans vis-à-vis de l'État. Cette grille de lecture éclaire-t-elle d'une certaine manière la lecture que vous faites d'une possible combinaison entre ethnicité et culture politique? Nombre d'analystes se sont fortement élevés contre le poids des cadres ethniques, jugés responsables de nombreux drames, et vous vous êtes vous-même opposé au tribalisme politique. Mais vous estimez aussi que le concept «d'ethnicité morale» pourrait être réellement utile pour édifier une culture civique. Pouvez-vous éclairer ces points?

8 J. L. : Tout provient, comme je l'ai mentionné plus haut, des faiblesses des économies et des États africains. En généralisant de manière outrancière, je dirais que l'une des principales caractéristiques de la culture politique africaine est le degré extraordinaire d'indépendance dont font preuve, ou auquel aspirent, la plupart des ménages africains dans l'organisation de leur existence. C'est surtout le cas en Afrique de l'Ouest : $75 \%$ des actifs y sont leurs propres employeurs. TC : C'est l'un des pourcentages les plus élevés au monde.

10 J. L.: Certainement. Ces pourcentages sont plus faibles en Afrique méridionale et orientale, mais il ne serait pas faux d'affirmer que le ménage africain moyen tend à être aussi autonome que possible, beaucoup plus qu'en Europe. Les ménages africains sont régis par une discipline efficace, imposée par le patriarcat qui définit le rôle des femmes et des enfants dans le système de production. Et je pense que la plupart des aspirations africaines tendent vers une telle indépendance familiale. J'ai autrefois étudié les définitions de la liberté chez les Kenyans. Tous mes interlocuteurs m'ont alors affirmé que disposer d'un grenier bien rempli, d'un cheptel en bonne santé, être capable de survivre à la famine et peut être d'aider des voisins en difficulté, cela représentait pour eux la définition universelle de ce qui fait la liberté.

11 L'indépendance est généralement la valeur socioculturelle la plus prisée, mais cette aspiration engendre un nombre croissant d'individus frustrés, en particulier des hommes et des femmes jeunes qui n'ont aujourd'hui ni accès à la terre, ni aux emplois, en raison d'une impressionnante croissance démographique depuis un siècle. Je pense que la croissance démographique africaine est la plus rapide de l'histoire mondiale, plus rapide encore que celle que connut la Chine au XIXe siècle, et qui plongea ce pays dans les troubles que l'on connaît. Cette aspiration à l'indé-pendance individuelle ou familiale est de plus en plus difficile à atteindre pour un nombre croissant de jeunes personnes, non intégrées, et de plus en plus perçues comme une menace. Ce sont ces jeunes gens qui deviennent les recrues potentielles des seigneurs de la guerre. Voilà donc une valeur universelle, l'indépendance, qui devient une source de mal-être, et même de danger.

12 Les Africains donnent du poids à l'indépendance familiale car ils savent avec acuité ce que c'est que d'être exploité, oppressé, non entendu, que de ne pouvoir faire confiance à leurs élites. Chacun en Afrique débat évidemment de ces questions, plus qu'en Europe peut-être, et les arènes naturelles où ces débats se déroulent sont avant tout les 
diverses aires linguistiques que compte chaque État africain. C'est au travers de ces discussions politiques et économiques qu'une communauté morale s'édifie, et c'est, à mon sens, un aspect totalement négligé de la genèse de l'ethnicité.

TC : Quelle lecture faites-vous de l'ethnicité africaine, dans son rapport au politique ?

14 J. L. : Il n'y a rien de particulièrement africain dans l'existence de l'ethnicité. Ce qui est tout à fait africain, en revanche, c'est qu'autant d'ethnies n'aient pas été réunies en entités plus larges, comme ce fut le cas en Europe au travers, il est vrai, de tragiques épreuves. $\mathrm{Au} \mathrm{Xx} \mathrm{X}^{\mathrm{e}}$ siècle, les guerres entre nations européennes, qui ont donné naissance aux guerres mondiales, ont établi des loyautés à un coût extrême ; ce n'est pas arrivé en Afrique, où le processus historique de formation de l'identité par des groupes de discussion politique fondés sur l'appartenance ethnique locale me paraît totalement différent. On ne peut donc s'attendre au même type de relations entre État et citoyenneté. On trouve en Afrique beaucoup plus d'échelles identitaires, de niveaux d'appartenances auxquels les États africains ont dû faire face, car ces identités ont été trop facilement manipulées. Ce que j'appelle «l'ethnicité morale » est un grand forum africain, un vaste espace de débats, qui ont le potentiel de faire naître une citoyenneté critique, mais qui sont aussi terriblement perméables aux manipulations opérées dans la distribution des bénéfices qui caractérise de nombreux États africains encore faibles.

TC : Vous avez aussi travaillé sur le problème des représentations de l'Afrique, et en particulier des perceptions fallacieuses ou ambiguës. Vous avez montré comment l'image de l'Afrique que véhiculent les médias populaires - un continent sans épaisseur historique, pauvre, sans espoir, - a un impact sur les politiques d'aide. Vous portez un jugement nuancé sur les grandes campagnes humanitaires des pop stars, telle Bob Geldof: elles sont certes un moyen efficace d'attirer l'attention sur l'urgence des problèmes de l'Afrique, mais elles réactualisent en un sens, dans un cadre postcolonial, la théorie populiste de la mission salvatrice de l'Occident, qui selon vous « infantilise les Européens tout comme les Africains ». Quant aux décideurs politiques, on a vu Tony Blair lancer la Commission pour l'Afrique en 2004, et même George W. Bush plaider pour un allégement de la dette. Quel jugement portez-vous sur la manière dont les principaux dirigeants du monde d'aujourd'hui s'attaquent aux problèmes africains? J. L.: On peut certes donner crédit à l'action humanitaire et aux décisions des politiques, mais il me semble qu'il existe une difficulté tout à fait centrale, et que personne ne peut résoudre pour l'heure : aux Nations unies, à l'Organisation mondiale du commerce, et dans toutes les structures internationales où se retrouvent les ministres des Affaires étrangères et les ministres du Commerce des pays européens, du Japon et des États-Unis, les dirigeants des pays riches disposent tous de cohortes de hauts fonctionnaires extrêmement informés et de véritables banques d'expertise, alors que les négociateurs africains n'ont quasiment aucune ressource comparable sur laquelle s'appuyer, pour développer face à leurs interlocuteurs un argumentaire efficace. L'inégalité en termes de savoir et de pouvoir de négociation est, disons-le, obscène, si j'en crois ceux qui ont l'expérience de ces rencontres. Un de mes anciens étudiants, devenu ambassadeur aux États-Unis, me disait qu'aucun pays Africain n'est en mesure d'agir efficacement à Washington, faute de contacts et de soutien bureaucratique suffisants.

17 TC : N'est-ce pas différent à l'ONU avec un secrétaire général africain? 
18 J. L. : C'est possible, mais je ne suis pas sûr de la force des délégations nationales aux Nations unies. Le secrétaire général fait preuve de compréhension et de sympathie, mais je ne sais à quel point il peut influencer les négociations pour remédier au déficit que j'ai évoqué. Qui plus est, l'inégalité dans le pouvoir de négociation dépend de bien des paramètres. Le principal défaut de l'approche des pop stars, des ONG, ou même des gouvernements européens ou autres, c'est que ces acteurs estiment que les gouvernements africains ne peuvent ou ne veulent rien faire, ou biaiseront l'aide au développement jusqu'à en annuler les effets par les pratiques de corruption. Je pense pour ma part que nos priorités devraient être d'identifier et de soutenir les Africains les plus doués, et disposant d'une certaine influence, afin de leur donner le soutien administratif et logistique dont ils ne peuvent disposer localement. Il faut aider les universités à former des négociateurs efficaces. Il faut prioritairement écouter les "voix africaines» qui sont non seulement authentiques, mais aussi tout à fait talentueuses, au moins autant que les nôtres.

TC : Cette nécessaire écoute des "voix africaines" renvoie à la question des perceptions défaillantes. Il existe aujourd'hui des dirigeants emblématiques dans le champ politique - Nelson Mandela, Ellen Johnson Sirleaf - ou dans celui de l'action sociale - la prix Nobel Wangari Matthai. Mais on pourrait tout autant citer de grands noms dans le domaine de la créativité, dans la littérature, les arts, le cinéma : des noms établis de longue date, ou de nombreuses figures émergentes. Peut-on dire que cette explosion de la créativité, manifeste en Afrique même mais aussi dans les grandes capitales culturelles mondiales, nous permette de considérer que les " voix africaines » sont plus entendues aujourd'hui qu'elles ne le furent jadis?

20 J. L. : Probablement, les « voix africaines » sont écoutées, dans ces domaines, et depuis longtemps : pensons au jazz, à Picasso. Mais je soupçonne qu'on les écoute sans se départir d'un certain degré d'aimables préjugés: les Africains sont supposés être naturellement des artistes, doués pour s'exprimer, proches des sources authentiques...

21 TC : N'est-ce pas différent en ce qui concerne la place que les Africains occupent maintenant dans la littérature?

22 J. L. : Oui, je pense que c'est le cas, et je connais le renouveau de la littérature africaine. Il y a de nombreux auteurs, particulièrement les Nigérians comme Wole Soyinka et Chinua Achebe, qui ont pris place dans le cursus des écoles anglaises. Ils sont largement connus, leurs textes sont enseignés dans les cours de littérature anglaise du Commonwealth de nos grandes universités, dont Cambridge. Mais je soupçonne néanmoins que la majorité des gens qui étudient ces formes de créativité africaine sont probablement des exilés, comme la plupart des créateurs africains. Cela confirme le fait malheureux qu'en Afrique, pour beaucoup, pour réussir il faut partir.

TC : C'est une forme de fuite des cerveaux?

J. L. : Exactement, il existe une fuite des créatifs.

$\mathrm{TC}$ : Peut-on dire que les récents succès africains, tant économiques que politiques, sont sinon ignorés, du moins trop peu commentés ? Au cours des dernières années, on a vu les taux de croissance s'élever, et plusieurs économies africaines sur la bonne voie : la Tanzanie, Madagascar, le Mozambique et d'autres. On a aussi vu un certain nombre d'évolutions politiques prometteuses, des élections démocratiques réussies. Y prête-ton suffisamment attention? Ces succès peuvent-ils aider à la transformation de l'Afrique et à celle de son image? 
26 J. L. : Vous avez raison. Les pays que vous avez mentionnés en Afrique orientale et méridionale s'en sortent très bien, particulièrement le Mozambique après les terribles inondations qu'il a connues voici quelques années. Il est également vrai que les transitions démocratiques, transparentes et pacifiques, sont de plus en plus fréquentes. En réalité, il semble difficile pour les médias généralistes de porter un regard neuf sur l'Afrique: c'est tellement plus simple de rabâcher les mêmes histoires. La presse britannique en particulier dispose de trop peu de correspondants en Afrique, et recourt à ceux que j'appelle les « journalistes parachutés » : en quarante-huit heures, ils voient ce qu'ils espéraient voir et confirment ce qu'ils pensaient savoir. Dans l'ensemble les médias renâclent à changer l'image de l'Afrique.

27 TC: En dépit des réussites que l'on vient d'évoquer et des avancées de la démocratisation, l'autoritarisme reste important dans de nombreux pays africains. $\mathrm{Ce}$ phénomène vous apparaît-il comme une séquelle des pratiques politiques coloniales et postcoloniales?

J. L. : En ce domaine, on ne prête pas assez attention aux différences grandissantes entre les pratiques politiques des différents pays africains. Entre ceux, encore rares, qui accordent les libertés civiques et disposent de gouvernements responsables, ceux dont les dirigeants restent des autocrates non repentis, et ceux qui ont sombré dans la guerre civile, les pays africains sont clairement divers. Voilà seulement une génération, ou un peu plus, que le colonialisme a pris fin en Afrique. Les cultures politiques ne peuvent s'améliorer profondément en si peu de temps. Certains pays ont pourtant fait bien mieux que d'autres, on ne le souligne pas assez. En somme, les pratiques politiques africaines postcoloniales ont acquis leur autonomie peu à peu, et elles diffèrent entre chaque pays. Le plus grand service que nous pouvons rendre à l'Afrique, c'est de prêter beaucoup plus d'attention à ces disparités accrues entre pays africains. Pourquoi trouve-t-on d'énormes différences entre deux voisins? Voyez la Somalie et le Kenya. La première n'a pas de gouvernement digne de ce nom. Le gouvernement kenyan, au contraire - et quoi qu'on en dise -, fonctionne presque comme Max Weber l'aurait souhaité. Le meilleur exemple que je connaisse à cet égard concerne le tsunami du 26 décembre 2004. L'ambassade américaine à Nairobi fut alertée par une station de l'US Navy dans l'océan Indien. Elle avertit le ministère de l'Intérieur kenyan. Une heure plus tard, la police évacuait les plages voisines de Mombasa. On ne déplora aucune victime : voilà un État fonctionnel !

29 TC : Vous appelez à prêter plus d'attention au passé, et à user au mieux de l'histoire pour créer un nouveau langage politique en Afrique. Vous soulignez ainsi la part de responsabilité des historiens, voire de la communauté scientifique dans son ensemble.

30 J. L. : Oui, bien sûr, j'écris en historien, et je suis surpris, et même choqué, de constater que l'on ne porte pas attention à la tradition critique propre aux Africains. Il est trop facile aux dirigeants autoritaires africains de dénoncer la démocratie et l'égalité comme des importations étrangères : c'est une trahison. Trop peu d'Africains sont assez au fait de leurs traditions pour dénoncer avec force cette imposture. Malgré leur professionnalisme, les historiens ont une part de responsabilité dans cette distance prise entre l'Afrique et ce que ses cultures historiques pourraient lui apporter.

31 TC : La France a été agitée, il y a quelques mois, par ce que certains journaux ont nommé la "question noire", entendons un débat sur le passé esclavagiste, sur la colonisation, mais aussi sur les difficultés d'intégration des migrants venus de nos anciennes colonies. Le débat a été exacerbé par une loi reconnaissant les aspects 
positifs de la colonisation, loi votée comme par inadvertance par le Parlement, puis retirée avant sa promulgation. La France éprouve des difficultés à penser son passé colonial et à gérer ses héritages postcoloniaux. La situation est-elle différente en Grande-Bretagne à cet égard?

32 J. L. : Le débat populaire britannique a été bien moins intense et moins informé qu'en France. On pourrait dire qu'à cet égard, le mythe national britannique se fonde sur l'idée que si les Britanniques ont bien pratiqué l'esclavage, ils ont aussi eu des héros qui ont œuvré à son abolition: au XIX ${ }^{\mathrm{e}}$ siècle, la Royal Navy tenta par tous les moyens de supprimer la traite en haute mer. On n'occulte pas l'esclavage, y compris dans les musées des grands ports de traite comme Bristol et Liverpool. Cela n'empêche pas un large consensus populaire qui considère que la faute esclavagiste fut ensuite expiée par l'Empire, perçu - pour autant que l'on se rappelle qu'il y eût un Empire ! - comme bienfaisant, porteur de l'éducation, de la santé, des pratiques de bon gouvernement... Une rare ignorance de notre histoire coloniale aujourd'hui réécrite est à la base de cette fierté populaire britannique, qui va jusqu'à imaginer que les déboires de l'Afrique tiennent à ce que Londres se soit retirée trop vite.

Quant à la gestion de l'héritage postcolonial, il est vrai que les communautés d'immigrants, qu'elles soient orientales, hindoues ou musulmanes, ou issues des Caraïbes ou d'Afrique occidentale, sont considérées comme ayant d'une certaine manière trouvé leur place dans une Grande-Bretagne de plus en plus multiraciale voire multiculturelle, où les immigrés peuvent se référer à des modèles «noirs ou bruns » ayant réussi dans le sport, la musique, la mode, les médias. Peut-être est-ce dû au fait que la GrandeBretagne a toujours été un état multinational, dans un sens où la France refuse de l'être. Les Écossais, les Irlandais et même les Cornouaillais se sont toujours vu accorder un certain degré d'autonomie nationale.

TC : Bien plus que les minorité provinciales en France !

J. L. : Question de valeurs, sans doute. Votre splendide insistance républicaine sur l'égalité n'a jamais fait partie de la culture politique britannique, et peut-être la Grande-Bretagne s'en est trouvée capable d'absorber plus facilement les nouveaux venus que la France.

TC : Pour terminer sur l'Afrique elle-même, on connaît le succès du concept d'afropessimisme. Pour ceux qui doutent qu'il suffise à définir la réalité africaine avec pertinence, verriez-vous un autre concept à lui opposer, pas nécessairement l'afrooptimisme, du reste...?

37 J. L. : Il y aurait beaucoup d'éléments à invoquer dans ce débat. J'aurais tendance à penser qu'il faut d'abord privilégier l'empathie, et réaliser que personne n'a de solution efficace aux problèmes qui se posent aujourd'hui en Afrique, en Europe ou ailleurs. La première étape pour parvenir à des relations saines avec l'Afrique, serait peut-être de l'aborder avec une certaine dose d'humilité. 


\section{NOTES}

1. John Lonsdale, "How to study Africa: From Victimhood to Agency ", OpenDemocracy. Voir : www.opendemocracy.net/democracy-africa_democracy/agency_2796.jsp. 1 septembre 2005; publié sous le titre «African Studies, Europe and Africa ", Afrika Spektrum, 40, 3, Hamburg, 2005, pp. 377-402.

\section{RÉSUMÉS}

Dans cet entretien accordé à Transcontinentales, ce grand nom de l'africanisme britannique remet en perspective les spécificités de l'histoire coloniale africaine qui aident à comprendre pourquoi les espoirs nourris à l'heure des indépendances ont été en partie déçus. Commentant les représentations convenues des médias et les ambiguïtés des grandes opérations humanitaires en faveur de l'Afrique, John Lonsdale appelle à reconnaître l'impérieuse nécessité d'œuvrer à un meilleur équilibre dans les pratiques de négociations portant sur l'Afrique, et souligne combien la redécouverte de l'histoire africaine pourra contribuer à nourrir une citoyenneté critique.

In this interview accorded to Transcontinentales John Lonsdale, one of the leading British historians of Africa, focuses on the particular characteristics of African colonial and post-colonial history that enable us to understand why the hopes that came with independence have been in part dampened. Commenting on conventional media representations and the ambiguity of largescale humanitarian operations aimed to benefit Africa, John Lonsdale appeals for recognition of the absolute necessity of working towards a better balance in negotiations concerning Africa, and emphasises how the rediscovery of African history may contribute to nurturing a discerning citizenry.

\section{INDEX}

Mots-clés : Histoire africaine, constructions nationales, images de l'Afrique

Keywords : African history, nation building, images of Africa 